\title{
Acceleration and escape of first cosmic rays
}

\author{
Yutaka Ohira ${ }^{* a}$ and Kohta Murase ${ }^{b c d e}$ \\ ${ }^{a}$ Department of Earth and Planetary Science, The University of Tokyo \\ ${ }^{b}$ Department of Physics, The Pennsylvania State University \\ ${ }^{c}$ Department of Astronomy \& Astrophysics, The Pennsylvania State University \\ ${ }^{d}$ Center for Particle and Gravitational Astrophysics, The Pennsylvania State University \\ ${ }^{e}$ Center for Gravitational Physics, Yukawa Institute for Theoretical Physics, Kyoto University \\ E-mail: v.ohiradeps.s.u-tokvo.ac.ip
}

\begin{abstract}
There are cosmic rays in the current universe. They play various important roles in the current universe. However, we do not understand when, where, how cosmic rays are first accelerated since the Big Bang. We show that supernova remnants of first stars can accelerate the first cosmic rays at $z \approx 20$. Shock waves of the first supernova remnants are nonrelativistic Weibel mediated shock, so that the coherent length scale of magnetic field fluctuations is much smaller than the gyroradius of the accelerated particles. Therefore, the maximum energy of the first CRs becomes much smaller than that in the current universe, which is about $100 \mathrm{MeV}$. Furthermore, we discuss cosmic-ray acceleration by accretion shocks due to the cosmological structure formation at $z \approx 20$. Since the accretion shocks at $z \approx 20$ propagate not an ionized medium but a neutral medium, they cannot accelerate the first cosmic rays.
\end{abstract}

36th International Cosmic Ray Conference -ICRC2019-

July 24th - August 1st, 2019

Madison, WI, U.S.A.

\footnotetext{
* Speaker.
} 


\section{Introduction}

There are many types of nonthermal particles in the current universe. We have a lot of evidences that the nonthermal particles are accelerated in various scales from the earth to clusters of galaxies. Cosmic rays (CRs) are one of the best examples. Since the pressure of CRs in our Galaxy is comparable to the thermal pressure, the CRs could even affect outflow dynamics of galaxies in the halo region, and their energy density is comparable to that of the warm-hot intergalactic medium [四].

Many observations of supernova remnants have shown the evidence that electrons and ions are accelerated to relativistic energies [ [2, B], 田]. Recently, a CR precursor, which is a prediction of the diffusive shock acceleration (DSA), was directly imaged []. Therefore, it is widely accepted that supernova remnants are the origin of galactic CRs in the current universe and the standard acceleration mechanism is DSA [ [6, 四].

However, it has not been studied when, where, and how the first CRs are accelerated since the Big Bang. We naively expect that shock waves in early universe accelerate the first CRs because CRs are accelerated by shock waves in the current universe. There are two energetic shock waves in early universe, supernova blast waves of the first stars and accretion shocks associated with the large scale structure formation. In this work, we consider particle accelerations by the two shock waves, showing that the first CRs are provided by shock waves driven by the first star explosion at $z \approx 20$. For more detailed arguments and calculations, see Ohira \& Murase (2018) [ [ ]]

\section{Shock waves driven by first-star explosions}

Cosmological simulations show that first stars are formed in dark matter halos with $M_{\mathrm{h}} \sim$ $10^{6} M_{\odot}$ at $z \approx 20$ [9]. Many theoretical studies show that the mass of the first stars becomes heavier than $10 M_{\odot}$ [ [0, [1], [2]]. The lifetime of the massive first stars is about Myr scale which is much shorter than the Hubble time at the age of the Universe $z \approx 20$. Therefore, the first stars gravitationally collapse, forming supernova remnants with strong shock waves at $z \sim 20$. The first stars emit a lot of ultraviolet photons until their gravitational collapse, which ionize surrounding gas and form HII regions. Kitayama et al. (2004)[[13] investigated the evolution of the HII region formed by the first stars and showed that the number density, temperature, and ionization fraction of the HII region are typically $n \sim 1 \mathrm{~cm}^{-3}, T \sim 1 \mathrm{eV}$, and $f_{\mathrm{i}}=1$, respectively.

Although some of the first stars may directly collapse to black holes and may not generate strong shock wave, their envelopes are ejected by a supernova explosion after the first stars gravitationally collapse. We here consider only normal core-collapse supernovae of the first stars, and the others are discussed in Ohira \& Murase (2018) [ [8]. The typical expansion velocity of the ejecta is given by

$$
u_{\mathrm{ej}} \simeq 4.5 \times 10^{8} \mathrm{~cm} \mathrm{~s}^{-1}\left(\frac{\mathscr{E}_{\mathrm{SN}}}{10^{51} \mathrm{erg}}\right)^{1 / 2}\left(\frac{M_{\mathrm{ej}}}{10 M_{\odot}}\right)^{-1 / 2},
$$

where $\mathscr{E}_{\mathrm{SN}}$ and $M_{\mathrm{ej}}$ are explosion energy and ejected mass, respectively. The shock velocity of the forward shock driven by the supernova is almost constant, $u_{\mathrm{sh}} \approx u_{\mathrm{ej}}$, during the free expansion phase, $t \lesssim t_{\mathrm{dec}}=\left(3 M_{\mathrm{ej}} / 4 \pi m_{\mathrm{p}} n\right)^{1 / 3} / u_{\mathrm{ej}}$, where $m_{\mathrm{p}}$ and $n$ are the proton mass and number density in the HII region. After $t>t_{\mathrm{dec}}$, the evolution of the shock velocity is given by $u_{\mathrm{sh}} \approx u_{\mathrm{ej}}\left(t / t_{\mathrm{dec}}\right)^{-3 / 5}$. 
The shock waves driven by the explosion of first stars propagate to the HII region ionized by the first stars. The shock wave becomes a collisionless shock because the dissipation mechanism of the shock waves is not due to the Coulomb collision. We first investigate what types of collsionless shock and magnetic field turbulence are generated in the first supernova remnants. Since the magnetic field strength in the HII region is expected to be too small to affect the collisionless shock [14], the HII region can be treated as an unmagnetized plasma. In that case, downstream hot plasma can leak to the shock upstream region, so that collisionless plasma instabilities are excited.

Since unmagnetized shocks with $u_{\mathrm{sh}} \approx 0.01 \mathrm{c}$ are not understood so far, we first consider evolution of two counter streaming electron-proton plasmas where the relative velocity is $u_{\mathrm{sh}} \sim 0.01 c$ and the temperature of each plasma is $T \sim 1 \mathrm{eV}$. The electron two-stream or Bunemann instabilities are the most unstable mode initially. After the saturation of those instabilities, electrons are heated to $T_{\mathrm{e}} \sim m_{\mathrm{e}} u_{\mathrm{sh}}^{2}$, but protons are still cold [ㅍ5]. Thereafter, the most unstable mode becomes ion-ion two stream instability and protons are heated to $T_{\mathrm{p}} \sim m_{\mathrm{e}} u_{\mathrm{sh}}^{2}$ [ए6]. After the saturation of the ion-ion two stream instability, the ion acoustic instability and the ion-ion two stream instability are stabilized, so that the ion Weibel instability becomes the most unstable mode, which generates magnetic field fluctuations. Since most of the kinetic energy of the proton beams are not dissipated by the early electrostatic instabilities, the ion Weibel instability mainly dissipates the proton beams. Therefore, collisionless shocks driven by the first star explosion are nonrelativistic Weibel mediated shocks. It has been shown by particle-in-cell simulations that relativistic Weibel mediated shocks can accelerate particles by DSA [ㅍ]. We can naively expect that first supernova remnant shocks can accelerate CRs at $z \approx 20$.

\section{Shock waves associated with the cosmological structure formation at $z \approx 20$}

In the current universe, accretion shocks of the cosmological structure formation have been considered as one of the high-energy accelerators in these environments [?]. Observations of the radio relic in the cluster of galaxies show that electrons are accelerated by the accretion shocks. However, the shock velocity of the accretion shock is expected to be slower at higher redshifts because halo masses that can collapse at $z \approx 20$ is much smaller than that in the current universe. The velocity of the accretion shock is about the virial velocity,

$$
u_{\mathrm{sh}} \approx v_{\mathrm{vir}}=6.8 \times 10^{5} \mathrm{~cm} \mathrm{~s}^{-1}\left(\frac{M_{\mathrm{h}}}{10^{6} M_{\odot}}\right)^{1 / 3}\left(\frac{1+z}{21}\right)^{1 / 2} .
$$

To accelerate CRs by the shock, about a half of matters around the accretion shock has to be ionized

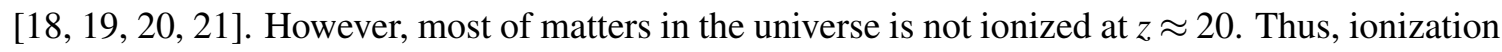
of the upstream medium is important for the particle acceleration at $z \approx 20$. For the first supernova remnant, ultraviolet photons from the first stars ionized their surrounding meida. On the other hand, the upstream medium of the accretion shock at $z \approx 20$ cannot be ionized because the shock velocity have to be larger than about $10^{7} \mathrm{~cm} / \mathrm{s}$ to ionize the upstream medium [L2]. Therefore, accretion shocks due to the cosmological structure formation at $z \approx 20$ propagate to neutral medium and cannot accelerate the first CRs. 


\section{Acceleration of the first CRs by the Weibel mediated nonrelativistic shock}

As discussed above, the first CRs would be accelerated by the first supernova remnants at $z \approx$ 20. Magnetic field fluctuations, $\delta B$, can be generated around the shock front of the first supernova remnants by the ion Weibel instability. Recent studies for the ion Weibel instability showed that the coherent length scale of the magnetic field fluctuations is $\lambda_{\delta \mathrm{B}}=\alpha c / \omega_{\mathrm{pp}}$ [23] , where $\alpha$ is a numerical factor. The magnetic field fluctuation scatter charged particles and some of the scattered particles are accelerated by DSA. Then, the acceleration time scale of DSA in the nonrelativistic Weibel mediated shock is given by (for derivation, see .Ohira \& Murase [ [8]])

$$
\begin{aligned}
& t_{\mathrm{acc}, \mathrm{p}} \approx \frac{40 \pi \hat{p}^{7}}{\alpha^{3} \eta_{\mathrm{p}}^{2} \beta_{\mathrm{sh}}^{8}} \omega_{\mathrm{pp}}^{-1} \quad \text { (for nonrelativistic protons) } \\
& t_{\mathrm{acc}, \mathrm{e}} \approx \frac{40 \pi \hat{p}^{14 / 3}}{\alpha^{3} \eta_{\mathrm{p}}^{2} \beta_{\mathrm{sh}}^{8}} \omega_{\mathrm{pp}}^{-1} \quad \text { (for relativistic electrons) },
\end{aligned}
$$

where $\hat{p}=p /\left(m_{\mathrm{p}} c\right) \ll 1$ and $m_{\mathrm{e}} / m_{\mathrm{p}} \ll \hat{p} \ll 1$ are assumed for protons and electrons, respectively. $\eta_{\mathrm{p}}$ is the ratio of the CR proton energy flux to the upstream kinetic energy flux in the shock rest frame, which is expected to be about 0.1 in the current universe.

The first CRs are most efficiently accelerated during the free expansion phase $t \lesssim t_{\text {dec }}$ because the acceleration time scale strongly depends on the shock velocity, $t_{\mathrm{acc}} \propto u_{\mathrm{sh}}^{-8}$. The time-limited maximum energy is almost the same as the size-limited maximum energy for the case of supernova remnants at $t=t_{\mathrm{dec}}[24,25]$. The maximum energies at the deceleration time are given by

$$
\begin{aligned}
& E_{\mathrm{max}, \mathrm{p}}^{\mathrm{dec}} \simeq 110 \mathrm{MeV}\left(\frac{\alpha}{10}\right)^{6 / 7}\left(\frac{\eta_{\mathrm{p}}}{0.1}\right)^{4 / 7}\left(\frac{M_{\mathrm{ej}}}{10 M_{\odot}}\right)^{-19 / 21}\left(\frac{\mathscr{E}_{\mathrm{SN}}}{10^{51} \mathrm{erg}}\right)\left(\frac{n}{1 \mathrm{~cm}^{-3}}\right)^{1 / 21} \\
& E_{\mathrm{max}, \mathrm{e}}^{\mathrm{dec}} \simeq 320 \mathrm{MeV}\left(\frac{\alpha}{10}\right)^{9 / 14}\left(\frac{\eta_{\mathrm{p}}}{0.1}\right)^{3 / 7}\left(\frac{M_{\mathrm{ej}}}{10 M_{\odot}}\right)^{-19 / 28}\left(\frac{\mathscr{E}_{\mathrm{SN}}}{10^{51} \mathrm{erg}}\right)^{3 / 4}\left(\frac{n}{1 \mathrm{~cm}^{-3}}\right)^{1 / 28}
\end{aligned}
$$

After the deceleration time, the shock velocity decreases with time, so that the size or time-limited maximum energy decreases with time,

$$
\begin{aligned}
& E_{\max , \mathrm{p}}=E_{\max , \mathrm{p}}^{\mathrm{dec}}\left(t / t_{\mathrm{dec}}\right)^{-38 / 35} \\
& E_{\max , \mathrm{e}}=E_{\max , \mathrm{e}}^{\mathrm{dec}}\left(t / t_{\mathrm{dec}}\right)^{-57 / 70} .
\end{aligned}
$$

For the parameters adopted at Eqs. (4.3) and (4.4), after $t=t_{\mathrm{c}} \simeq 29 t_{\mathrm{dec}}$, the maximum energy of protons is limited by the Coulomb loss and the maximum energy at $t=t_{\mathrm{c}}$ becomes $E_{\mathrm{max}, \mathrm{p}} \sim$ $2.9 \mathrm{MeV}$. Therefore, the first supernova remnants can scatter CR protons in the energy ranges $3 \mathrm{MeV} \lesssim E \lesssim 100 \mathrm{MeV}$ to the intergalactic medium.

\section{Summary}

We studied the acceleration and escape of the first CRs in this work. Our results can be summarized as follows: 1) The first CRs can be accelerated by the first supernova remnants driven by the explosion of first stars at $z \approx 20.2$ ) The first CRs are accelerated by DSA in the nonrelativistic Weibel mediated shocks. 3) The maximum energy of the first CRs is about $100 \mathrm{MeV}$. 4) The first 
$\mathrm{CR}$ protons with energies larger than a few $\mathrm{MeV}$ can escape from the first supernova remnants. 5) Accretion shocks of the cosmological structure formation at $z \approx 20$ cannot accelerate particles because the shocks propagate in neutral media.

\section{Acknowledgments}

This work is supported by JSPS KAKENHI Grant Number JP16K17702 (Y.O.), JP19H01893(Y.O.) and Alfred P. Sloan Foundation and NSF Grant No. PHY-1620777 (K.M.). Y.O. is supported by MEXT/JSPS Leading Initiative for Excellent Young Researchers.

\section{References}

[1] K. Murase and M. Fukugita, Energetics of high-energy cosmic radiations, PRD, 99, 063012, 2019

[2] K. Koyama et al., Evidence for shock acceleration of high-energy electrons in the supernova remnant SN1006, Nature, 378, 255, 1995

[3] Y. Ohira, K. Murase, and R. Yamazaki, Gamma-Rays from Molecular Clouds Illuminated by Cosmic Rays Escaping from Interacting Supernova Remnants, MNRAS, 410, 1577, 2011

[4] M. Ackermann et al., Detection of the Characteristic Pion-Decay Signature in Supernova Remnants, Science, 339, 807, 2013

[5] Y. Ohira and Yamazaki, Inverse Compton emission from a cosmic-ray precursor in RX J1713.7-3946, JHEAp, 13, 17, 2017

[6] A. R. Bell, The Acceleration of Cosmic Rays in the Shock Fronts-I, MNRAS, 182, 147, 1978

[7] R. D. Blandford, and J. P. Ostriker, Particle Acceleration by Astrophysical Shocks, ApJ, 221, L29, 1978

[8] Y. Ohira and K. Murase, Origin of the First Cosmic Rays, arXiv:1812.02950

[9] N. Yoshida, T. Abel, L. Hernquist, and N. Sugiyama, Simulations of Early Structure Formation: Primordial Gas Clouds, ApJ, 592, 645, 2003

[10] K. Omukai and N. Nishi, Formation of Primordial Protostars, ApJ, 508, 141, 1998

[11] T. Hosokawa, K. Omukai, N. Yoshida, and H. W. Yorke Protostellar Feedback Halts the Growth of the First Stars in the Universe, Science, 334, 1250, 2011

[12] S. Hirano, et al., One Hundred First Stars: Protostellar Evolution and the Final Masses, ApJ, 781, 60, 2014

[13] T. Kitayama, N. Yoshida, H. Susa, and M. Umemura, The Structure and Evolution of Early Cosmological H II Regions, ApJ, 613, 645, 2004

[14] K. Doi and H. Susa, Generation of a Seed Magnetic Field around First Stars: The Biermann Battery Effect, ApJ, 714, 93, 2011

[15] Y. Ohira and F. Takahara, Absence of Electron Surfing Acceleration in a Two-Dimensional Simulation, ApJ, 661, L171, 2007

[16] Y. Ohira, and F. Takahara, Oblique Ion Two-Stream Instability in the Foot Region of a Collisionless Shock, ApJ, 668, 320, 2008 
[17] A. Spitkovsky, Particle Acceleration in Relativistic Collisionless Shocks: Fermi Process at Last?, ApJ, 682, L5, 2008

[18] Y. Ohira, Effects of Leakage Neutral Particles on Shocks, ApJ, 758, 979, 2012

[19] Y. Ohira, Simulations of Collisionless Perpendicular Shocks in Partially Ionized Plasmas, PRL, 111, 245002, 2013

[20] Y. Ohira, Magnetic Field Amplification by Collisionless Shocks in Partially Ionized Plasmas, ApJ, 817, 137, 2016

[21] Y. Ohira, Injection to Rapid Diffusive Shock Acceleration at Perpendicular Shocks in Partially Ionized Plasmas, ApJ, 827, 36, 2016

[22] M. A. Dopita, L. M. Krauss, R. S. Sutherland, C. Kobayashi, and C. H. Lineweaver Re-ionizing the universe without stars, Ap\&SS, 335, 345, 2011

[23] C. Ruyer and F. Fiuza, Disruption of Current Filaments and Isotropization of the Magnetic Field in Counterstreaming Plasmas, PRL, 120, 245002, 2018

[24] Y. Ohira, K. Murase, and R. Yamazaki, Escape-limited model of cosmic-ray acceleration revisited, $A \& A$ 513, A17, 2010

[25] Y. Ohira, R. Yamazaki, N. Kawanaka, K. Ioka, Escape of cosmic-ray electrons from supernova remnants, MNRAS 427, 91, 2012 